\title{
Factores obstétricos claves en los resultados neonatales y a los dos años de seguimiento en la prematuridad extrema
}

\author{
Nayara López C. 1, Mar González A. 1, Laura Álvarez C. 2, Nuria Martínez S. 1 , \\ Antonio González G. 1 , Félix Omeñacá T. 2 , Belén San José V. a \\ ${ }^{1}$ Servicio de Obstetricia y Ginecología, ${ }^{2}$ Servicio de Pediatría y Neonatología, Hospital Universitario La Paz, Madrid, \\ España.
}

a Bioestadística.

\section{RESUMEN}

Objetivo: Analizar la morbimortalidad en pretérminos extremos evaluando la influencia de factores obstétricos. Método: Estudio retrospectivo de 132 casos nacidos entre las semanas 23 y 27 en el Hospital La Paz, desde 2003 a 2005. Se establecieron tres grupos obstétricos: Amenaza de Parto Pretérmino, Rotura Prematura de Membranas y la asociación de ambas. Se evaluaron como variables obstétricas: tocolisis, corticoterapia, motivo de finalización de la gestación y vía de parto, y como variables en niños: mortalidad y morbilidad respiratoria, neurológica, visual y auditiva en neonatos y a los dos años. Resultados: Los casos con amenaza de parto pretérmino presentaron mayor displasia broncopulmonar y ductus arterioso persistente que los otros dos grupos obstétricos $(p=0,03)$. Las pacientes con amenaza de parto pretérmino y tocolisis desarrollaron menos hemorragia intraventricular $[36,4 \%(12 / 33)]$ e infarto periventricular (0\%) que los casos sin tocolisis, en los que aparecieron en el 68,4\% (13/19) y 31,6\%(6/19), respectivamente ( $p=0,03$ y $p=0,001)$. Además en este subgrupo, los casos que recibieron corticoterapia desarrollaron menos infarto periventricular (0\%) y parálisis cerebral a los 2 años [6,7\% (2/30)], que los que no la recibieron, en los que apareció un $40 \%(6 / 15)$ de infarto y un $40 \%(4 / 10)$ de parálisis cerebral, respectivamente $(p=0,0001$ y $\mathrm{p}=0,02)$. La hemorragia intraventricular y la parálisis cerebral fueron más frecuentes en partos vaginales de casos con amenaza de parto pretérmino que en cesáreas $[63,3 \%(19 / 30)$ y $26,1 \%(6 / 23)$ frente a $27,3 \%$ (6/22) y $0 \% ; p=0,01$ y 0,03$]$. Conclusión: La conducta obstétrica puede modificar el pronóstico neonatal y a los 2 años de seguimiento.

\section{PALABRAS CLAVE: Prematuridad extrema, amenaza de parto pretérmino, rotura prematura de membranas, corticoterapia, tocolisis}

\section{SUMMARY}

Objective: Analyse morbidity and mortality in extreme preterm at birth and at 2 year follow-up evaluating the influence of obstetrical factors. Methods: Retrospective study of 132 cases born between weeks 23 and 27 at La Paz Hospital from 2003 to 2005. Three obstetrical groups were established: Threat of Preterm Birth, Premature Rupture of Membranes and the combination of both. The following were evaluated as obstetrical variables: tocolysis, corticosteroid therapy and type of delivery. As variables in children: mortality and respiratory, neurological, visual and auditive morbidity in neonates and two years of age. Results: In the cases of threat of preterm birth a greater bronchopulmonary dysplasia and persistent ductus arteriosus appeared than in the other two obstetrical groups $(p=0.03)$. Focusing on the threat of birth group, the cases with maternal tocolysis developed fewer neurological complications, intraventricular hemorrhage of $36.4 \%(12 / 33)$ 
and periventricular infarct of $0 \%$, whereas the cases without tocolysis showed $68.4 \%(13 / 19)$ and $31.6 \%$ $(6 / 19)$ respectively $(p=0.03, p=0.001)$. Also in this subgroup, the cases that received corticosteroid therapy developed less periventricular infarct $(0 \%)$ and cerebral palsy at age $2[6.7 \%(2 / 30)]$ than the ones that did not receive it in which the percentages were $40 \%(6 / 15)$ and $40 \%(4 / 10)$ ( $p=0.0001$ and $p=0.02$ respectively). Also, intraventricular hemorrhage and cerebral palsy were more frequent in vaginal delivery than in caesarean sections in this subgroup [63.3\% (19/30) and $26.1 \%(6 / 23)$ against $27.3 \%(6 / 22)$ and $0 \% ; p=0.01$ and $\mathrm{p}=0.03)]$. Conclusion: Obstetrical characteristics and behaviour can have a decisive impact in the neonatal outcome and after two-year follow-up.

\section{KEY WORDS: Extreme prematurity, threat of preterm delivery, premature rupture of membranes, corticosteroid therapy, tocolysis}

\section{INTRODUCCIÓN}

La tasa de neonatos extremadamente prematuros se ha visto incrementada desde hace unos años, en parte debido al desarrollo de las técnicas de reproducción asistida y a la mayor edad de las pacientes gestantes (1). En España, el registro oficial de recién nacidos de $<28$ semanas fue de $0,75 \%$ en 2006 (1).

La prematuridad constituye un problema crucial en la actualidad, no sólo por su incidencia, sino también por ser una situación clínica que conlleva importantes decisiones obstétricas y neonatales, debiendo ser tratado de forma conjunta por obstetras y pediatras para conseguir resultados perinatales óptimos.

La prematuridad se asocia con un tercio de todas las muertes en niños, y es responsable del $45 \%$ de casos de parálisis cerebral, del $35 \%$ de trastornos visuales y del $25 \%$ de casos de retraso cognitivo y déficit auditivo (2).

Los avances alcanzados gracias a los corticoides prenatales y a la optimización de los cuidados neonatales han permitido una disminución de las edades gestacionales consideradas como límite de la viabilidad. Así la supervivencia en pretérminos extremos ha aumentado considerablemente $(3,4)$. Sin embargo, el objetivo clave es alcanzar la máxima supervivencia pero con mínima morbilidad neonatal y a largo plazo $(5,6,7)$.

Existen pocos estudios con datos de la evolución de los pretérminos extremos, especialmente con resultados en el seguimiento a medio-largo plazo. La existencia de resultados perinatales es imprescindible para la toma de decisiones antes, durante y después del nacimiento. Sólo así se podrá adoptar una estrategia individualizada y ofrecer información detallada a los padres, especialmente entre las 23 y 25 semanas, donde los aspectos éticos deben ser tenidos en cuenta cautelosamente (derecho de los neonatos a la vida, a la no maleficencia y a la justicia y el derecho de los padres a decidir sobre sus hijos) (8). A partir de la semana 25 existe acuerdo en adoptar una conducta activa $(9,10)$.

El objetivo del estudio fue analizar la morbilidad y la mortalidad en pretérminos extremos, al nacimiento y a los dos años de seguimiento, evaluando la influencia de factores obstétricos (tocolisis, corticoterapia y vía de parto) en dichos resultados.

\section{MATERIAL Y MÉTODO}

Estudio observacional retrospectivo de 132 casos nacidos entre las semanas $23+0$ y $27+6$ de gestación (edad gestacional confirmada por datación ecográfica) en el Hospital Universitario La Paz desde enero de 2003 a diciembre de 2005.

El estudio fue realizado en coordinación por obstetras y pediatras y bajo las consideraciones éticas marcadas por el hospital.

Se ha considerado la edad gestacional y no el peso como criterio de inclusión para desestimar el error introducido por el crecimiento intrauterino retardado que incluiría en el mismo grupo niños de muy diferente grado de madurez. La edad gestacional datada ecográficamente es un parámetro claramente objetivo y exacto.

La edad gestacional seleccionada (entre las semanas $23+0$ y $27+6$ de gestación) se incluiría en el grupo establecido de parto extremadamente prétermino.

Se establecieron tres grupos obstétricos: Amenaza de Parto Pretérmino (APP), Rotura Prematura de Membranas (RPM) y Amenaza de Parto Pretérmino con Rotura Prematura de Membranas (APPRPM).

Como amenaza de parto pretérmino se incluyeron dos subgrupos sin signos clínicos de corioamnionitis: pacientes con dinámica uterina y mo- 
dificaciones cervicales (dilatación de $<2 \mathrm{~cm}$ con borramiento $\leq 70 \%$ ) y casos de verdadera APP, con dinámica regular y cérvix completamente borrado y $\geq 2 \mathrm{~cm}$ de dilatación.

Los criterios de corioamnionitis clínica considerados fueron la presencia de fiebre $>37,8^{\circ} \mathrm{C}$ con leucocitosis $>15.000 / \mathrm{ml}$ y desviación izquierda o con PCR elevada y en ascenso.

La rotura prematura de membranas se definió por la visualización directa de salida de líquido amniótico o por resultado positivo del test ACTIM PROM en casos dudosos y ausencia de sangre.

En casos con APP se valoró el tipo, la tocolisis con atosibán y el motivo de finalización de la gestación y en todas las pacientes se estudiaron la corticoterapia y la vía de parto.

La tocolisis fue realizada con atosibán $6,75 \mathrm{mg}$ intravenoso en bolo, seguido de $18 \mathrm{mg} /$ hora durante 3 horas y después se mantuvo una infusión intravenosa $6 \mathrm{mg} /$ hora durante 45 horas. La tocolisis se inició ante la presencia de modificaciones cervicales desde el ingreso hasta la reevaluación 2 horas después y en casos de verdadera APP.

Se consideró corticoterapia a la administración de dos dosis intramusculares de betametasona 12 mg intramuscular cada 12 horas.

La elección de la vía de parto no estuvo marcada por la edad gestacional, sino por la vía más rápida de parto en casos de APP no frenable.

Desde el punto de vista neonatal se analizaron: mortalidad, displasia broncopulmonar (DBP), ductus arterioso persistente (DAP), enterocolitis necrotizante (NEC), hemorragia intraventricular (HIV), infarto y leucomalacia periventricular, retinopatía de la prematuridad (ROP), sepsis y anemia. A los dos años se evaluaron: datos somatométricos, parálisis cerebral, retraso motor y cognitivo, hiperreactividad bronquial, defectos de refracción y alteraciones auditivas.

La displasia broncopulmonar se definió como la necesidad de oxígeno suplementario por encima de las 36 semanas de edad postconcepcional. El ductus arterioso persistente fue diagnosticado mediante ecocardiografía con Doppler en niños con datos clínicos sugerentes como soplo sistólico de eyección, precordio hiperactivo, taquicardia, pulsos saltones en región postductal o empeoramiento respiratorio. La enterocolitis necrotizante se definió por la presencia de un estadio de Bell mayor o igual a II (signos y síntomas clínicos con neumatosis intestinal en las radiografías) y, en grado III, con perforación intestinal inminente o manifiesta. Las ecografías cerebrales seriadas desde los dos primeros días de vida hasta las 40 semanas de edad postconcepcional definieron las alteraciones cerebrales (hemorragia intraventricular, infarto y leucomalacia periventricular). La valoración de la retinopatía de la prematuridad se llevó a cabo mediante la realización de fondo de ojo. La sepsis se confirmó bacteriológicamente con la presencia de un hemocultivo positivo.

Se realizaron revisiones periódicas desde los 15 días tras el alta con periodicidad trimestral durante el primer año y semestral durante el segundo. El diagnóstico de parálisis cerebral se estableció sobre la base de la presencia de alteraciones motoras espásticas no progresivas. El coeficiente de desarrollo fue determinado por un psicólogo a los 2 años de edad corregida, utilizando la escala de Brunet y Lezine y/o de Bayley, para el retraso motor y cognitivo y estableciéndose el diagnóstico con puntuaciones por debajo de la media (11). La audición se evaluó mediante la realización de potenciales auditivos evocados a todos los niños entre los 3 y 6 meses de edad corregida.

Estudio descriptivo: para variables cuantitativas continuas se utilizó la media junto con la desviación estándar y para las cualitativas, frecuencias absolutas y relativas expresadas en porcentaje.

Estudio univariante: las comparaciones entre variables cuantitativas continuas se realizaron mediante pruebas no paramétricas ( $U$ de Mann-Whitney) y entre variables cualitativas mediante la prueba de Chi cuadrado $\left(x^{2}\right)$ y test exacto de Fisher.

El valor de $p<0,05$ indicó la significación estadística.

\section{RESULTADOS}

La edad gestacional media al nacimiento fue de $25,6 \pm 1,4$ semanas. Se excluyen 27 fetos que murieron intraútero. La historia neonatal se obtuvo en 105 neonatos y el seguimiento a los 2 años en 83 niños. No hubo casos de malformaciones.

El 76,2\% (80/105) fueron gestaciones únicas y el $23,8 \%(25 / 105)$ gestaciones gemelares.

El 49,5\% (52/105) de pacientes presentaron amenaza de parto pretérmino, el 14,3\% (15/105) rotura prematura de membranas y el $36,2 \%(38 / 105)$ amenaza de parto pretérmino con rotura prematura de membranas.

Los casos de APP presentaron una estancia hospitalaria media de 1,0 $\pm 1,3$ días, frente a 4,0 \pm 6,3 días en pacientes con RPM y a 3,0 $\pm 3,4$ días en casos de APP-RPM ( $p=0,008)$.

Estas diferencias se hallaron concretamente entre los casos de APP y los de RPM $(p=0,03)$, y entre las pacientes con APP y APP-RPM $(p=0,04)$. La administración de corticoides intraútero fue diferente en los tres grupos. La recibieron el $71 \%$ 
(37/52) de neonatos y el $75 \%(30 / 40)$ de los niños con seguimiento a los dos años en casos de APP, el $87 \%(13 / 15)$ de casos neonatales y el $91 \%(10 / 11)$ de casos a dos años en pacientes con RPM y el 95\% (36/38) de neonatos y el $97 \%$ (31/32) de niños con seguimiento a los dos años en casos de APP$\operatorname{RPM}(p=0,01)$.

La semana de gestación media al parto fue de $25,7 \pm 1,2$ para casos de APP, de 26,0 $\pm 0,9$ para RPM y de $26,1 \pm 1,1$ para APP-RPM $(p=0,1)$.

La vía de parto fue vaginal en el 59,6\% (31/52) de casos con APP, en el 20\% (3/15) con RPM y en el $42,1 \%(16 / 38)$ en pacientes con APP y RPM $(p=0,018)$.

La morbimortalidad estratificada por grupo obstétrico se detalla en la Tabla I.

Variables de Amenaza de Parto Pretérmino (APP): Dentro de los subgrupos considerados bajo el término de APP, se observaron diferencias en la edad gestacional al parto. En pacientes con dinámica uterina asociada a modificaciones cervicales la edad gestacional media fue de $26,4 \pm 0,8$ semanas y en casos de verdadera APP (>2cm) de 25,2 $\pm 1,1$ semanas $(p=0,0001)$.

En el $82,6 \%$ (43/52) de los casos el motivo de finalización de la gestación fue la amenaza de parto pretérmino no frenable. En estos casos de parto pretérmino inminente la gestación se finalizó a las $25,5 \pm 1,1$ semanas, frente a las $27,3 \pm 0,4$ semanas en casos de finalización por otros motivos $(p=0,01)$.

La edad gestacional media al nacimiento fue de $26,1 \pm 1,1$ semanas para las cesáreas y de 25,4 \pm 1,2 para la vía vaginal $(p=0,04)$. Recibieron corticoides intraútero el $66,7 \%(20 / 30)$ de los fetos nacidos por vía vaginal y el $78 \%(17 / 22)$ de nacidos por cesárea $(p=0,2)$.

La influencia de la tocolisis, la corticoterapia y la vía de parto en la morbi-mortalidad neonatal y a los dos años de seguimiento se describe en las Tablas II, III y IV. Los casos con tocolisis desarrollaron menos hemorragia intraventricular $[36,4 \%(12 / 33)$ e infarto periventricular $(0 \%)$ ] que los casos sin tocolisis, en los que aparecieron en el $68,4 \%$ (13/19) y $31,6 \%(6 / 19)$, ( $p=0,03$ y $p=0,001$, respectivamente). Los casos que recibieron corticoterapia desarrollaron menos infarto periventricular $(0 \%)$ y parálisis cerebral a los 2 años [6,7\% (2/30)] que los que no la recibieron, en los que apareció un 40\% (6/15) de infarto y un $40 \%(4 / 10)$ de parálisis cerebral ( $p=0,0001$ y $p=0,02$, respectivamente). La hemorragia intraventricular y la parálisis cerebral fueron más frecuentes en partos vaginales que en cesáreas $[63,3 \%(19 / 30)$ y $26,1 \%(6 / 23)$ frente a $27,3 \%(6 / 22)$ y $0 \%$, respectivamente $(p=0,01$ y 0,03$)]$.

Tabla I

MORBIMORTALIDAD SEGÚN GRUPO OBSTÉTRICO

\begin{tabular}{lcccc}
\hline Variables & APP (52) & RPM (15) & APP+RPM (38) & Valor p \\
\hline Mortalidad & $12(23,1 \%)$ & $4(26,7 \%)$ & $6(15,8 \%)$ & 0,59 \\
Displasia broncopulmonar & $25(48,1 \%)$ & $5(33,3 \%)$ & $8(21,1 \%)$ & 0,03 \\
Enteroclitis necrotizante & $2(3,8 \%)$ & $3(20 \%)$ & $3(7,9 \%)$ & 0,11 \\
Ductus arterioso persistente & $37(71,2 \%)$ & $6(40 \%)$ & $19(50 \%)$ & 0,03 \\
Anemia & $44(84,6 \%)$ & $12(80 \%)$ & $34(89,5 \%)$ & 0,64 \\
Sepsis & $33(63,5 \%)$ & $8(53,3 \%)$ & $26(68,4 \%)$ & 0,58 \\
Retinopatía & $26(50 \%)$ & $6(40 \%)$ & $15(39,5 \%)$ & 0,56 \\
Hemorragia intraventricular & $25(48,1 \%)$ & $6(40 \%)$ & $13(34,2 \%)$ & 0,41 \\
Infarto periventricular & $6(11,5 \%)$ & 0 & $1(2,6 \%)$ & 0,13 \\
Parálisis cerebral a 2 años & $6(15 \%)$ & $1(9,1 \%)$ & $2(6,3 \%)$ & 0,48 \\
Retraso motor a 2 años & $12(30 \%)$ & $3(27,3 \%)$ & $8(25 \%)$ & 0,89 \\
Retraso cognitivo a 2 años & $12(30 \%)$ & $5(45,5 \%)$ & $7(21,9 \%)$ & 0,32 \\
Defecto refracción a 2 años & $8(20 \%)$ & $2(18,2 \%)$ & $3(9,4 \%)$ & 0,45 \\
Defecto auditivo a 2 años & $19(47,5 \%)$ & $6(54,5 \%)$ & $17(53,1 \%)$ & 0,85 \\
Peso p<10 a 2 años & $14(50 \%)$ & $6(66,7 \%)$ & $12(44,4 \%)$ & 0,51 \\
Hiperreactividad bronquial a 2 años & $14(35 \%)$ & $4(36,4 \%)$ & $12(37,5 \%)$ & 0,97
\end{tabular}

RPM: Rotura Prematura de Membranas. APP: Amenaza de Parto Pretérmino. 
Tabla II

RELACIÓN ENTRE ADMINISTRACIÓN DE TOCOLISIS CON ATOSIBÁN Y PATOLOGÍAS NEONATALES EN CASOS DE AMENAZA DE PARTO PRÉTERMINO

\begin{tabular}{lccc}
\hline Variables & Tocolisis Sí & Tocolisis NO & Valor p \\
\hline Mortalidad & $18,2 \%(6 / 33)$ & $31,6 \%(6 / 19)$ & 0,3 \\
Displasia broncopulmonar & $45,5 \%(15 / 33)$ & $52,6 \%(10 / 19)$ & 0,7 \\
Entecolitis necrotizante & $6,1 \%(2 / 33)$ & $0 \%$ & 0,5 \\
Ductus arterioso persistente & $72,2 \%(24 / 33)$ & $68,4 \%(13 / 19)$ & 0,7 \\
Anemia & $81,8 \%(27 / 33)$ & $89,5 \%(17 / 19)$ & 0,6 \\
Sepsis & $60,6 \%(20 / 33)$ & $68,4 \%(13 / 19)$ & 0,7 \\
Retinopatía de prematuridad & $48,5 \%(16 / 33)$ & $52,6 \%(10 / 19)$ & 1,0 \\
Hemorragia intraventricular & $36,4 \%(12 / 33)$ & $68,4 \%(13 / 19)$ & 0,04 \\
Infarto periventricular & $0 \%$ & $31,6 \%(6 / 19)$ & 0,001 \\
Leucomalacia periventricular & $3 \%(1 / 33)$ & $5,3 \%(1 / 19)$ & 1,0 \\
Parálisis cerebral a 2 años & $7,4 \%(2 / 27)$ & $30,8 \%(4 / 13)$ & 0,07 \\
Retraso motor a 2 años & $25,9 \%(7 / 27)$ & $38,5 \%(5 / 13)$ & 0,4 \\
Retraso cognitivo a 2 años & $29,6 \%(8 / 27)$ & $30,8 \%(4 / 13)$ & 1,0 \\
Defecto refracción a 2 años & $22,2 \%(6 / 27)$ & $15,4 \%(2 / 13)$ & 1,0 \\
Defecto auditivo a 2 años & $40,7 \%(11 / 27)$ & $61,5 \%(8 / 13)$ & 0,3 \\
Hiperreactividad bronquial a 2 años & $40,7 \%(11 / 27)$ & $23,1 \%(3 / 13)$ & 0,3 \\
Peso percentil<10 a 2años & $55 \%(15 / 27)$ & $38,4 \%(5 / 13)$ & 0,6 \\
\hline
\end{tabular}

Tabla III

RELACIÓN ENTRE ADMINISTRACIÓN DE CORTICOIDES Y PATOLOGÍAS NEONATALES Y A LOS DOS AÑOS DE SEGUIMIENTO EN CASOS DE AMENAZA DE PARTO PRÉTERMINO

\begin{tabular}{lccc}
\hline Variables & Corticoides Sí & Corticoides NO & Valor p \\
\hline Mortalidad & $18,9 \%(7 / 37)$ & $33,3 \%(5 / 15)$ & 0,2 \\
Displasia broncopulmonar & $54,1 \%(20 / 37)$ & $33,3 \%(5 / 15)$ & 0,2 \\
Enterocolitis necrotizante & $5,4 \%(2 / 37)$ & $0 \%$ & 1,0 \\
Ductus arterioso persistente & $78,4 \%(29 / 37)$ & $53,3 \%(8 / 15)$ & 0,09 \\
Anemia & $86,5 \%(32 / 37)$ & $80 \%(12 / 15)$ & 0,6 \\
Sepsis & $67,6 \%(25 / 37)$ & $53,3 \%(8 / 15)$ & 0,3 \\
Retinopatía de prematuridad & $54,1 \%(20 / 37)$ & $40 \%(6 / 15)$ & 0,5 \\
Hemorragia intraventricular & $43,2 \%(16 / 37)$ & $60 \%(9 / 15)$ & 0,3 \\
Leucomalacia periventricular & $5,4 \%(2 / 37)$ & $0 \%$ & 1,0 \\
Infarto periventricular & $0 \%$ & $40 \%(6 / 15)$ & 0,0001 \\
Parálisis cerebral 2 años & $6,7 \%(2 / 30)$ & $40 \%(4 / 10)$ & 0,02 \\
Retraso motor a 2 años & $30 \%(9 / 30)$ & $30 \%(3 / 10)$ & 1,0 \\
Retraso cognitivo a 2 años & $30 \%(9 / 30)$ & $30 \%(3 / 10)$ & 1,0 \\
Defecto refracción a 2 años & $23,3 \%(7 / 30)$ & $10 \%(1 / 10)$ & 0,6 \\
Defecto auditivo a 2 años & $46,7 \%(14 / 30)$ & $50 \%(5 / 10)$ & 1,0 \\
Peso percentil<10 a 2 años & $56,6 \%(17 / 30)$ & $0 \%$ & 0,09 \\
\hline
\end{tabular}




\section{Tabla IV}

EVALUACIÓN DEL PAPEL DE LA VÍA DE PARTO EN EL DESARROLLO DE LAS PATOLOGÍAS ESTUDIADAS EN CASOS DE AMENAZA DE PARTO PRÉTERMINO

\begin{tabular}{lllc}
\hline Variables & Parto vaginal & Cesárea & Valor p \\
\hline Mortalidad & $23,3 \%(7 / 30)$ & $22,7 \%(5 / 22)$ & 1,0 \\
Displasia broncopulmonar & $53,3 \%(16 / 30)$ & $40,9 \%(9 / 22)$ & 0,4 \\
Enterocolitis necrotizante & $0 \%(0 / 30)$ & $9,1 \%(2 / 22)$ & 0,1 \\
Ductus arterioso persistente & $66,7 \%(20 / 30)$ & $77,3 \%(17 / 22)$ & 0,5 \\
Anemia & $93,3 \%(28 / 30)$ & $72,7 \%(16 / 22)$ & 0,05 \\
Transfusión neonatal & $90 \%(27 / 30)$ & $63,6 \%(14 / 22)$ & 0,03 \\
Sepsis & $66,7 \%(20 / 30)$ & $59,1 \%(13 / 22)$ & 0,7 \\
Retinopatía de la prematuridad & $30 \%(15 / 30)$ & $59,1 \%(13 / 22)$ & 0,7 \\
Hemorragia intraventricular & $63,3 \%(19 / 30)$ & $27,3 \%(6 / 22)$ & 0,01 \\
Leucomalacia periventricular & $6,7 \%(2 / 30)$ & $0 \%(0 / 22)$ & 0,5 \\
Infarto periventricular & $16,7 \%(5 / 30)$ & $4,5 \%(1 / 22)$ & 0,2 \\
Parálisis cerebral a 2 años & $26,1 \%(6 / 23)$ & $0 \%(0 / 22)$ & 0,03 \\
Retraso motor a 2 años & $30,4 \%(7 / 23)$ & $29,4 \%(5 / 17)$ & 1,0 \\
Retraso cognitivo a 2 años & $34,8 \%(8 / 23)$ & $23,5 \%(4 / 17)$ & 0,5 \\
Defecto de refracción a 2 años & $8,7 \%(2 / 23)$ & $35,3 \%(6 / 17)$ & 0,05 \\
Alteraciones auditivas a 2 años & $47,8 \%(11 / 23)$ & $47,1 \%(8 / 17)$ & 1,0 \\
Hiperreactividad bronquial a 2 años & $21,7 \%(5 / 23)$ & $52,9 \%(9 / 17)$ & 0,05 \\
Peso < percentil 10 a 2 años & $30 \%(7 / 23)$ & $70.5 \%(12 / 17)$ & 0,1 \\
\hline
\end{tabular}

Variables de Rotura Prematura de Membranas (RPM): El 20\% (3/15) de neonatos nacieron por vía vaginal y el $80 \%(12 / 15)$ por cesárea. En los casos con seguimiento a los dos años, hubo un $9 \%(1 / 11)$ de partos vaginales y un $91 \%(10 / 11)$ de casos de cesáreas. Recibieron corticoides intraútero el 100\% (3/3) de los fetos nacidos por vía vaginal, y el 83,3\% (10/12) de nacidos por cesárea $(p=1,0)$. La edad gestacional media al nacimiento fue de $26,4 \pm 0,8$ semanas para las cesáreas y de $24,7 \pm 0,5$ para la vía vaginal $(p=0,01)$.

La relación entre la administración de corticoides y las patologías neonatales y a los dos años de seguimiento, se detalla en la Figura 1. En la Figura 2 se muestra el papel de la vía de parto en el desarrollo de las patologías estudiadas en neonatos.

En el seguimiento a los dos años se demostró que el $90 \%$ de los fetos que no desarrollaron parálisis cerebral fueron casos de cesárea. El retraso motor y cognitivo se observó en su totalidad en el grupo de cesáreas (3/3) y (5/5), respectivamente. También el $100 \%$ de casos en los que apareció déficit de refracción y alteración auditiva, al igual que hiperreactividad bronquial, se halló en cesá- reas (2/2) y (6/6) y (4/4), respectivamente. El $100 \%$ de los fetos nacidos mediante cesárea alcanza un peso $\geq$ percentil $10(3 / 3)$.

Variables de Amenaza de Parto Pretérmino y Rotura Prematura de Membranas (APP-RPM): La edad gestacional media al nacimiento fue de $26,1 \pm 1,1$ semanas para casos nacidos por cesárea y de 26,0 $\pm 1,1$ para nacidos por vía vaginal $(p=0,6)$. Se obtuvieron 16 casos de parto vaginal y 21 casos de cesárea entre los neonatos y hubo 15 casos de parto vaginal y 16 casos de cesárea entre los niños con seguimiento a los dos años. El 93,8\% (15/16) de los fetos nacidos por parto vaginal recibieron corticoides intraútero, frente al 95,5\% (21/22) de nacidos por cesárea $(p=1,0)$. Recibieron tocolisis con atosiban 32 de los 38 casos $(84,2 \%)$.

En las Figuras 3 y 4 se evalúa la distribución de patologías en pacientes con corticoterapia y la influencia de la vía de parto en las diversas enfermedades.

El infarto periventricular se encontró en el $50 \%$ $(1 / 2)$ de fetos sin maduración y en ningún caso con corticoterapia $(p=0,05)$. 


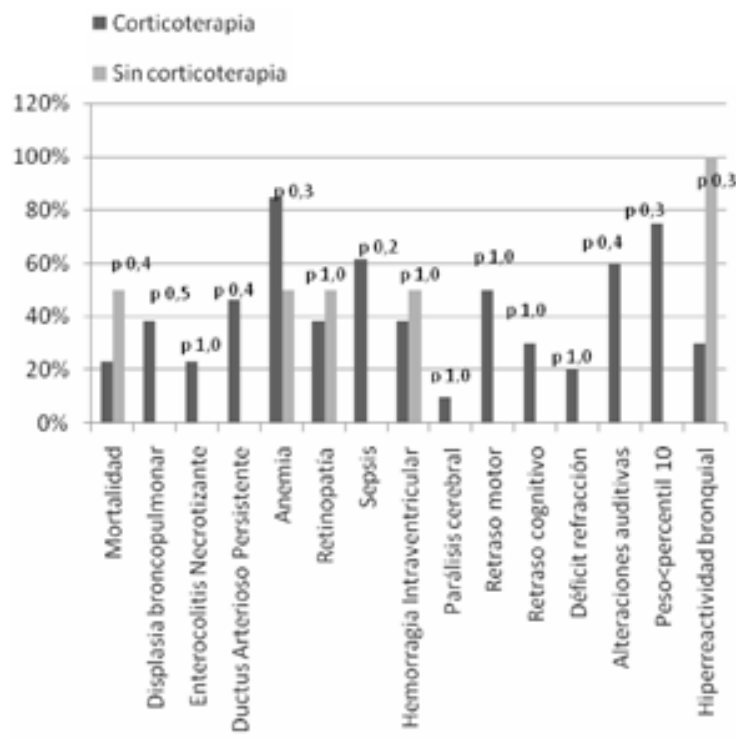

Figura 1. Relación entre la administración de corticoides y las patologías estudiadas en neonatos y a los 2 años de seguimiento en casos de RPM.

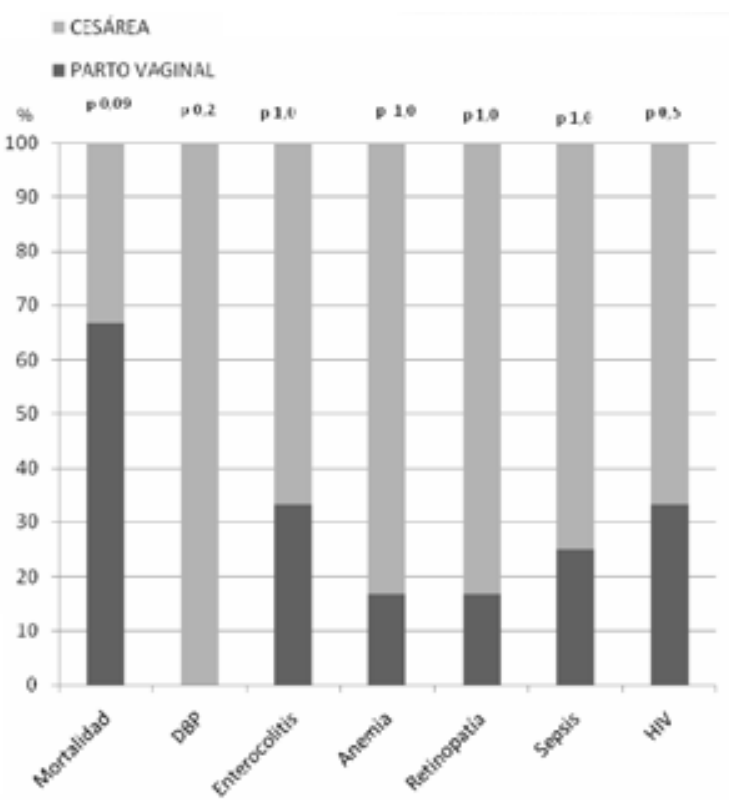

Figura 2. Evaluación del papel del la vía de parto en el desarrollo de las patologías estudiadas en casos de RPM (DBP: Displasia broncopulmonar. HIV: Hemorragia intraventricular).

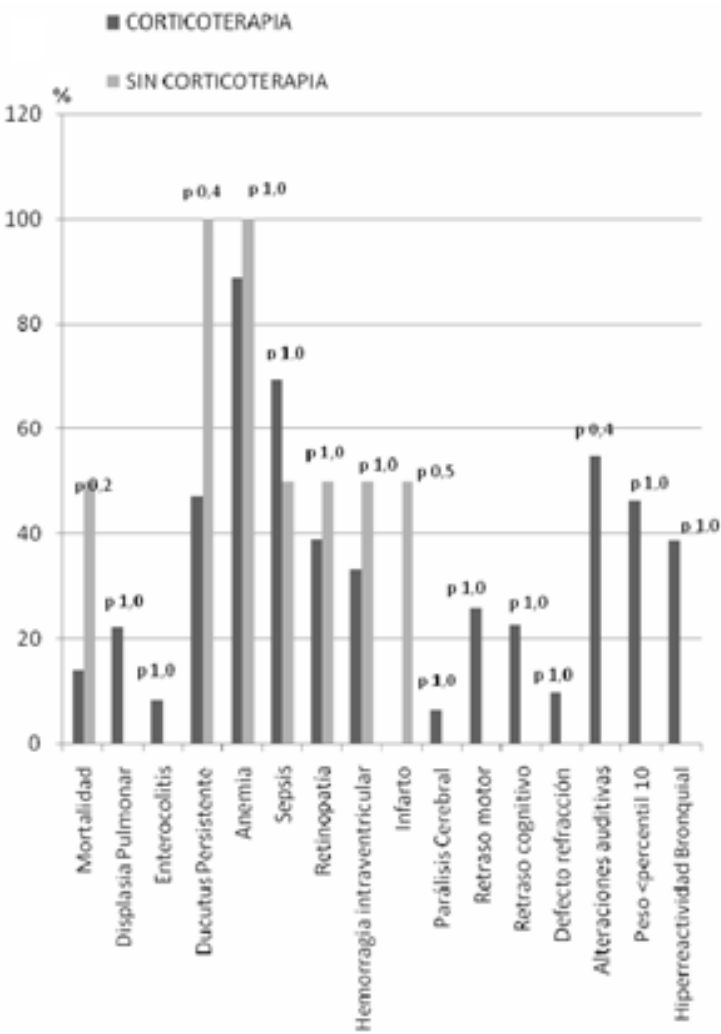

Figura 3. Relación entre la administración de corticoides y las patologías estudiadas en neonatos y a los 2 años de seguimiento en casos de RPM y APP.

\section{DISCUSIÓN}

Se han valorado las situaciones obstétricas más frecuentes en la práctica clínica (amenaza de parto pretérmino y/o rotura prematura de membranas) como escenario materno inicial intentando descifrar su implicación en los resultados perinatales. El grupo de rotura prematura de membranas se subdividió porque en el día a día se presentan pacientes que rompen la bolsa a edades gestacionales tempranas, sin que ello se acompañe de dinámica de parto, manteniendo esta situación durante semanas. Los datos presentados mostraron que, a edades gestacionales tan precoces, la rotura de la bolsa y el desencadenamiento del parto sólo estaban separados por unos pocos días.

Los tres grupos obstétricos presentaron una evolución clínica diferente. Las pacientes con APP, al presentar una estancia hospitalaria media desde el ingreso hasta el parto bastante menor que los 


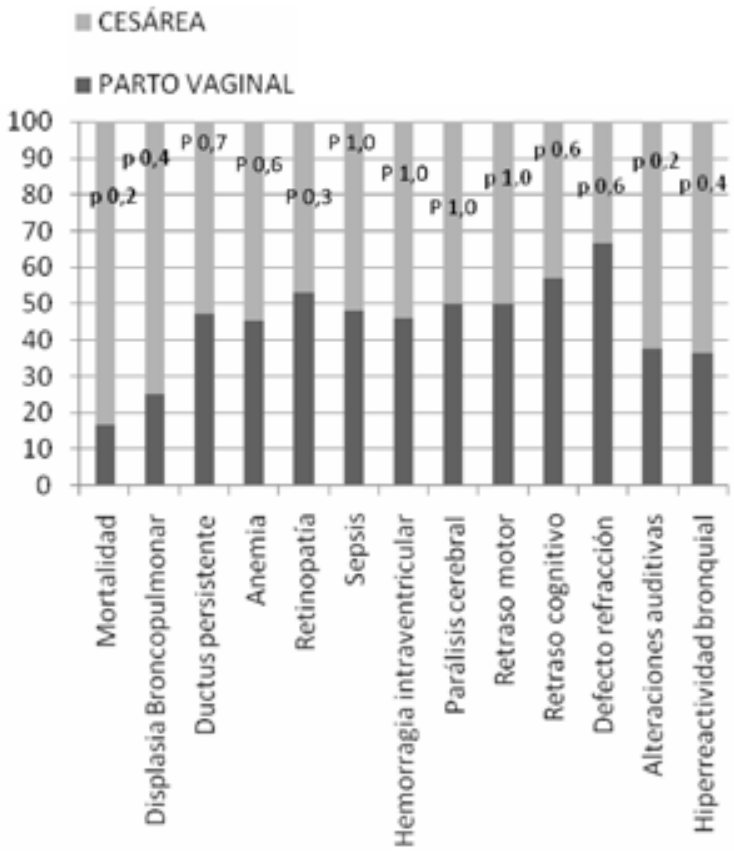

Figura 4. Desarrollo de patología neonatal y a los dos años de seguimiento en función de la vía de parto en pacientes con RPM Y APP.

otros dos grupos, se pudieron beneficiar de menos terapias obstétricas. Así, la mayoría de los casos de RPM y de APP-RPM recibieron corticoterapia intraútero durante su estancia hospitalaria y la mayor parte de las pacientes con APP-RPM pudieron recibir también tocolisis.

Entre las pacientes con APP hubo más casos de displasia broncopulmonar y ductus arterioso persistente que entre las pacientes con RPM o APP-RPM. La displasia broncopulmonar, junto con la retinopatía y el daño cerebral se han considerado factores adversos en el neurodesarrollo posterior de los neonatos $(12,13,14)$. Sin embargo, no se hallaron diferencias en los resultados neurológicos entre los diferentes grupos obstétricos.

La edad gestacional media al parto en casos con APP fue ligeramente menor, al igual que el número de pacientes a las que se administró corticoterapia. Los corticoides antenatales son eficaces en reducir la enfermedad de membrana hialina, la displasia broncopulmonar y la mortalidad y, también presentan un efecto positivo en la hemorragia intraventricular, el ductus arterioso persistente y la enterocolitis necrotizante (15). Sólo el beneficio sobre el infarto periventricular y la parálisis cerebral quedan demostrados con los resultados obtenidos.
Entre los tres grupos también hubo diferencias en la vía de parto, siendo más prevalente la vía vaginal en los casos de APP. Así, la edad gestacional y, sobre todo, la vía de parto vaginal y la menor corticoterapia pudieron ejercer su influencia, al menos en parte, en los resultados descritos. Otros factores como la corioamnionitis subclínica también podrían tener un papel relevante.

Entre los casos de amenaza de parto pretérmino, el pronóstico neurológico se vio empobrecido en la vía vaginal (hemorragia intraventricular y parálisis cerebral a los 2 años), obteniéndose también más casos de anemia. Qiu y cols (16), aportan datos de daño cerebral en la vía vaginal, aunque sin llegar a parálisis cerebral, mientras que Anderson y cols (17) no encuentra mayor tasa de hemorragia cerebral entre los partos vaginales pero sí mayor progresión a grado 3 y 4 en casos de hemorragia intraventricular. Hay varios factores que podrían influir en estos resultados. Por una parte, la edad gestacional media en partos vaginales, y además en casos de APP no frenable, fue menor que en cesáreas. La edad gestacional al parto se ha propuesto como marcador de morbilidad neonatal pudiendo explicarse, al menos parcialmente, de este modo la diferencia entre las vías de parto $(18,19)$. Por otro lado, aunque sin llegar a ser estadísticamente significativo, el número de casos con corticoterapia entre los partos vaginales fue inferior al de cesáreas por lo que los fetos nacidos mediante cesárea se beneficiarían del efecto positivo neurológico de los corticoides (15). Tanto los casos de APP que recibieron tocolisis como los casos a los que se administró corticoterapia presentaron menos complicaciones neurológicas. Las pacientes que recibieron tocolisis presentaron menos hemorragia intraventricular e infarto periventricular y en los fetos que recibieron corticoterapia hubo menos casos de infarto periventricular y parálisis cerebral. Probablemente el efecto beneficioso de la tocolisis se deba al hecho de permitir prolongar la gestación 48 horas para la administración de corticoides $(20,21)$.

\section{CONCLUSIÓN}

La tocolisis, la corticoterapia y la vía de parto pueden modificar el pronóstico neonatal y a los 2 años de seguimiento.

\section{BIBLIOGRAFÍA}

1. Rellan S, García C, Aragón M. El recién nacido prematuro. Protocolos AEPED.

2. Mandy GT. Short-term complications of the premature infant. UpToDate. Last review version 19.2: mayo 2011. 
3. Johnson S, Wolke D, Marlow N; Preterm Infant Parenting Study Group. Developmental assessment of preterm infants at 2 years: validity of parent reports. Dev Med Child Neurol 2008;50:58-62.

4. Washburn LK, Dillard RG, Goldstein DJ, Klinepeter $K L$, deRegnier RA, O'Shea TM. Survival and major neurodevelopmental impairment in extremely low gestational age newborns born 1990-2000: a retrospective cohort study. BMC Pediatr 2007;7:20.

5. Bode MM, D'Eugenio DB, Forsyth N, Coleman J, Gross CR, Gross SJ. Outcome of extreme prematurity: a prospective comparison of 2 regional cohorts born 20 years apart. Pediatrics 2009;124:866-74.

6. de Kleine MJ, den Ouden AL, Kollée LA, Ilsen A, van Wassenaer AG, Brand R, Verloove-Vanhorick SP. Lower mortality but higher neonatal morbidity over a decade in very preterm infants. Paediatr Perinat Epidemiol 2007;21:15-25.

7. Jankova J, Dimitrov A. Extreme prematurity--medical and social problem. Akush Ginekol (Sofiia) 2009;48:31-7.

8. Vohr BR, Wright LL, Poole WK. Neurodevelopmental outcomes of extremely low birth Weight infants 32 week gestation between 1993 and 1998. Pediatrics 2005; 116:635-43.

9. Mercurio MR. The ethics of newborn resuscitation. Semin Perinatol 2009;33:354-63.

10. Pignotti MS, Donzelli G. Perinatal care at the threshold of viability: an international comparison of practical guidelines for the treatment of extremely preterm births. Pediatrics 2008;121:e193-8.

11. Leversen KT, Sommerfelt K, Rønnestad A, Kaaresen PI, Farstad T, Skranes J, Støen R, Elgen IB, Rettedal $S$, Eide GE, Irgens LM, Markestad T.Predicting neurosensory disabilities at two years of age in a national cohort of extremely premature infants. Early Hum Dev 2010;86:581-6.
12. Schmidt B, Asztalos EV, Roberts RS, Robertson CM, Sauve RS, Whitfield MF. Impact of bronchopulmonary dysplasia, brain injury, and severe retinopathy on the outcome of extremely low-birth-weight infants at 18 months. JAMA 2003;289:1124-9.

13. Laughon M, O'Shea MT, Allred EN et al. ELGAN Study Investigators. Chronic lung disease and developmental delay at 2 years of age in children born before 28 weeks' gestation. Pediatrics 2009;124:637-48.

14. Doyle LW, Anderson PJ. Pulmonary and neurological follow-up of extremely preterm infants. Neonatology 2010;97:388-94.

15. Magny JF, Rigourd V, Kieffer F, Voyer M. Perinatal corticosteroid therapy: modalities, efficacy, consequences. J Gynecol Obstet Biol Reprod (Paris) 2001;30:36-46.

16. Qiu H, Paneth N, Lorenz JM, Collins M. Labor and delivery factors in brain damage, disabling cerebral palsy, and neonatal death in low-birth-weighy infants. Am J Obstet Gynecol 2003;189:1143-9.

17. Anderson GD, Bada HS, Shaver DC et al. The effect of cesarean section on intraventricular hemorrhage in the preterm infant. Am J Obstet Gynecol 1992;166:1091-9.

18. Fellman V, Hellström-Westas L, Norman M et al. Oneyear survival of extremely preterm infants after active perinatal care in Sweden. JAMA 2009;301:2225-33.

19. Wood NS, Marlow N, Costeloe K, Gibson AT, Wilkinson AR. Neurologic and developmental disability after extremely preterm birth. EPICure Study Group. N Engl J Med 2000;343:378-84.

20. Di Renzo GC, Al Saleh E, Mattei A, Koutras I, Clerici G. Use of tocolytics: what is the benefit of gaining 48 hours for the fetus? BJOG 2006;113 Suppl 3:72-7.

21. Di Renzo GC, Donati Sarti R, Gori F, Gerli S. Tocolysis: which are the benefits? Minerva Ginecol 2006;58:479-87. 